\title{
Response analysis of an accelerating unbalanced rotating system with both random and interval variables
}

\author{
Chao Fu ${ }^{1,2 \dagger}{ }^{\dagger}$, Yuandong Xu${ }^{1}$, Yongfeng Yang ${ }^{2}$, Kuan $\mathrm{Lu}^{2}$, Fengshou Gu${ }^{1}$, Andrew Ball ${ }^{1}$ \\ ${ }^{1}$ Centre for Efficiency and Performance Engineering, University of Huddersfield, \\ Huddersfield, HD1 3DH, United Kingdom \\ ${ }^{2}$ Institute of Vibration Engineering, Northwestern Polytechnical University, Xi'an 710072, P.R. China
}

\begin{abstract}
This paper investigates the accelerating up transient vibrations of a rotor system under both the random and uncertain-but-bounded uncertainties. The Polynomial Chaos Expansion (PCE) coupled with the Chebyshev surrogate method is used to analyses the propagations of the two categorizes of uncertainties. The output responses will possess the characteristics of both bounded quantities and statistical moments. As a hybrid non-intrusive uncertainty quantification (UQ) procedure, the deterministic rotor model is taken as a black box and will only be executed at specific parameter points. A number of uncertain physical parameters are studied and the corresponding transient responses are presented. The accuracy and efficiency are verified by the Monte Carlo simulations (MCS) in combination with the scanning scheme and also other hybrid analysis framework. It will provide guidance for the accurate transient dynamic analysis of engineering problems with hybrid uncertainties.
\end{abstract}

Keywords: transient rotordynamics; hybrid uncertainties; polynomial chaos expansion; interval analysis

\section{Introduction}

Rotating component is an important and fundamental class of motion element in modern machineries. Transient vibration behaviours of rotor systems are of great significance for the condition monitoring and dynamical investigations [1, 2]. Darpe et al. [3] investigated the transient responses of a cracked rotor and discussed the diagnosis of crack fault. Choy and Padovan [4] analyzed the nonlinear transient vibrations of a rubbing rotor. Insightful rub mechanism and relevant dynamic behaviours were studied. Recently, Yue et al. [5] used the start-up accelerating response for dynamical balancing of turbine engine rotors. Uncertain factors such as wear and load variation are present in engineering mechanical systems $[6,7]$ and their dynamics will be affected by the uncertainties $[8,9]$. The sources and causes can be diverse, as explained in [10]. Researchers have paid their attention to the uncertain vibrations of systems with crack fault $[11,12]$ and the instabilities under uncertainty [13]. There are practical demands from both the academic and industrial communities for robust evaluations of the transient rotordynamics under uncertainty, which calls for the non-deterministic analyses.

The uncertainties in an engineering problem can be generally described by two groups of variables, i.e. the random $[14,15]$ and interval ones $[16,17]$. The two categories correspond to the probabilistic and non-probabilistic representations. Random uncertainties are used to describe those whose precise

† Corresponding author. E-mail addresses: fuchao0606@mail.nwpu.edu.cn, C.Fu@hud.ac.uk (C. Fu); F.Gu@hud.ac.uk (F. Gu). 
probability density functions (PDFs) are already known. Many sophisticated applications [18-22] of the stochastic methods to rotordynamics can be found, including the principle of maximum entropy, the MCS, the global optimization method [23] and the perturbation method [24]. The PDF of a random quantity can be constructed from sufficient statistical information. It is suggested to use probabilitybased descriptions when there are plenty of samples or verified empirical distribution models, because they are precise and accurate for uncertainty propagation analysis. The mathematical deduction is rigorous as well. Direct application of the MCS may lead to prohibitive computational burden due to its poor convergence rate. Perturbation methods are subject to small uncertainty limitations. Some procedures require necessary amendments to the existing solution codes, which is featured as intrusive. The PCE, which is one of the spectral methods, can work in non-intrusive way and exhibits excellent performance in stochastic modeling of random variables and processes. It is popular and widely employed in the UQ for mechanical systems recently. Researchers adapted the PCE to the stochastic dynamics analyses of rotor systems [25-27] and further investigated the influences of the PC order on the responses of an asymmetric rotor near resonances [28]. In order to avoid subjective results, interval variables characterized only by their bounds are alternatively adopted in situations where the precise PDFs of uncertainties are unavailable. Small sample sizes with inadequate information to summarize such distribution models are common in early design stages. Moreover, quantities changing with time such as degradation or simply insufficient knowledge of the designers can also lead to such conditions. Uncertainty analysis involving interval quantities can be carried out using direct interval arithmetic, which may, however, cause large overestimations known as the wrapping effect. Intrusive interval methods such as the interval Taylor approach and perturbation method $[29,30]$ can often get relatively tight response bounds, but they are difficult to adapt to different problems as the mathematic formulation is complex and modifications should be made to the original solvers. The Chebyshev inclusion function (CIF) [31], which controls well the overestimations, has the advantages of transparency and simplicity in concept. It is non-intrusive and applicable to different kinds of dynamic problems governed by ODEs and PDEs as it treats them as a black box. A collocation scheme for the interval method [32] was further proposed to alleviate the increasing computational efforts in multi-dimensional cases. Early efforts have been made by researchers using the interval methods to the UQ for the dynamics of rotating systems [33-37]. However, the non-probabilistic interval methods have not yet aroused sufficient attention in the field of rotordynamics, although some fruitful findings were presented.

In engineering context, problems with partial incomplete information are often confronted and both the random and interval uncertainties are contained at the same time [38-40]. There is a rising number of hybrid uncertainty studies in statics, structural dynamics and reliability analysis [41-44]. In the field of rotordynamics, most of the researchers concentrated on random type uncertainty analysis and a few on the interval UQ. A likelihood-based approach was proposed to study the effects of the mixed uncertainties on the dynamics of a rubbing Jeffcott rotor system [45]. The transient dynamics of rotor systems under hybrid uncertainties has aroused inadequate attention and remain unrevealed. It will pose challenges for the UQ algorithms to achieve accurate estimations in the attenuation area of transient 
vibrations where the fluctuations are present. In this paper, we are devoted to investigate the transient dynamics of a rotor system during the accelerating up process with both the random and interval uncertainties included. A non-intrusive dual-layer uncertainty propagation model is proposed to cope with the two categories of uncertain parameters, which overcomes the constraints of small-range uncertainties and the symbolic manipulations in the existing algorithms. To improve the efficiency necessary strategies aimed at reducing the underlying computational costs are incorporated in cases with multiple uncertain parameters. Investigation into the influence mechanisms of different uncertain parameters on the transient vibrations will be carried out.

The content of the rest of this paper is as follows. First, a hollow-shaft overhung rotor system and its governing time-varying motion equations are described. Second, the representations of the two types of uncertainties and the underlying problem to be solved are explained. The non-intrusive uncertainty handling methodologies are then illustrated in detail. Third, the numerical results obtained are compared with those provided by the classic sampling methods. Finally, the transient vibration responses are demonstrated through numerical simulations with multiple uncertain parameters.

\section{Rotating system modeling}

The rotating system considered in this paper has a typical overhung configuration, which has wide applications in jet engines and pumps. The schematic diagram is presented in Fig.1. It consists of a stepdiameter hollow rotating shaft, two isotropic elastic supports and a rigid disk placed at the right side of the rotor. The length of the rotor is $L$ and the disk has a mass $m_{d}$. Considering the feature of the system configuration, the transfer matrix method is convenient here for the deduction of the motion equations. Unlike the finite element method, it keeps the sizes of the modeling matrices constant regardless of the number of degrees of freedom, which is beneficial for program computation. At the cross-section of a station in $x$ direction, the motion status can be characterized by four parameters $[x \beta M Q]^{\mathrm{T}}$, i.e. lateral displacement, bending angle, bending moment and shear force. The same applies to the $y$ direction. Every feature component such as the shaft, bearing and disk should be modeled as a station and their transfer modes are predefined. When utilizing the transfer matrix method, the rotor in Fig. 1 is discretized into the two bearing elements, one disk element and six beam elements (Each shaft is divided into three elements). The cross-section status relationships of a rigid disk are shown in Fig. 2, where $\beta_{i}$ is the angle between the shaft centerline and the horizontal line [46]. Due to unbalance on the mass disk and gravity, the excitation forces can be expressed as

$$
\left\{\begin{array}{l}
F_{x}=m e\left[\omega^{2} \cos (\omega t+\phi)+\dot{\omega} \sin (\omega t+\phi)\right] \\
F_{y}=m e\left[\omega^{2} \sin (\omega t+\phi)-\dot{\omega} \cos (\omega(t) t+\phi)\right]+m g
\end{array}\right.
$$

where $\omega$ and $\dot{\omega}$ denotes the rotating speed and the angular acceleration of the system, $m$ is the mass of the disk. Notation $e$ is the eccentricity and $\phi$ represents the initial unbalance angle. The moments attached to the system due to the gyroscopic effect are

$$
\left\{\begin{array}{l}
M_{x}=I_{d} \ddot{\beta}+I_{p} \omega \dot{\alpha} \\
M_{y}=I_{d} \ddot{\alpha}-I_{p} \omega \dot{\beta}
\end{array}\right.
$$


in which $\beta, \alpha$ are the angles of the disk in the two perpendicular planes, $I_{p}$ and $I_{d}$ are the polar and transverse moments of inertia. The increments of the status parameters when passing the disk are

$$
\Delta \mathbf{S}=\left[0,0, \Delta M_{x}, \Delta Q_{x}, 0,0, \Delta M_{y}, \Delta Q_{y}\right]^{\mathrm{T}}
$$

with

$$
\begin{cases}\Delta M_{x}=M_{x}, & \Delta Q_{x}=-m \ddot{x}-c \dot{x}+F_{x} \\ \Delta M_{y}=M_{y}, & \Delta Q_{y}=-m \ddot{y}-c \dot{y}+F_{y}\end{cases}
$$

Therefore, the status vector before the disk $\mathbf{S}_{b}$ and after the disk $\mathbf{S}_{a}$ have the following relationship

$$
\mathbf{S}_{a}=\Delta \mathbf{S}+\mathbf{S}_{b}
$$

For a beam element, the status vector relationship in the $x$ direction is illustrated in Fig. 3. Considering these elements, the tansfer function is expressed as

$$
\mathbf{S}_{b}=\mathbf{T}_{s 2} \mathbf{T}_{b 2} \mathbf{T}_{s 1} \mathbf{T}_{b 1} \mathbf{S}_{0}
$$

in which $\mathbf{T}_{s 2}, \mathbf{T}_{b 2}, \mathbf{T}_{s 1}$ and $\mathbf{T}_{b 1}$ are the transfer matrices for shaft 2, bearing 2, shaft 1 and bearing 1, respectively. They all have the same size $8 \times 8 . \mathbf{S}_{0}$ is the status vector of the left-end cross section of the rotor system. The matrix expressions for beam elements and bearing elements can be found in Ref. [47]. The boundary conditions should be used to derive the motion equations. To create free boundaries, vitual additional shafts (transfer matrix $\mathbf{T}_{v}$ ) with a very small length are added to both ends of the rotor. The influences to the system can be neglected. The following expressions hold true

$$
\mathbf{S}_{R}=\mathbf{T}_{v} \mathbf{S}_{b}, \mathbf{S}_{0}=\mathbf{T}_{v} \mathbf{S}_{L}
$$

where $\mathbf{S}_{L}$ and $\mathbf{S}_{R}$ are the status vector of the new left and right ends of the rotor. In $\mathbf{S}_{L}$ and $\mathbf{S}_{R}$, the shear force $Q$ and bending moment $M$ should equal to zero

$$
\left[\begin{array}{ll}
\mathbf{T}(3: 4,1: 2) & \mathbf{T}(3: 4,5: 6) \\
\mathbf{T}(7: 8,1: 2) & \mathbf{T}(7: 8,5: 6)
\end{array}\right]\left[\begin{array}{c}
x \\
\beta \\
y \\
\alpha
\end{array}\right]_{L}+\left[\begin{array}{cc}
\mathbf{T}_{v}(3: 4,3: 4) & \mathbf{T}_{v}(3: 4,7: 8) \\
\mathbf{T}_{v}(7: 8,3: 4) & \mathbf{T}_{v}(7: 8,7: 8)
\end{array}\right]\left[\begin{array}{c}
\Delta M_{x} \\
\Delta Q_{x} \\
\Delta M_{y} \\
\Delta Q_{y}
\end{array}\right]=\left[\begin{array}{l}
0 \\
0 \\
0 \\
0
\end{array}\right]
$$

with $\mathbf{T}=\mathbf{T}_{v} \mathbf{T}_{s 2} \mathbf{T}_{b 2} \mathbf{T}_{s 1} \mathbf{T}_{b 1} \mathbf{T}_{v}$. Subscript $L$ represents the left side of the rotor. Let $\mathbf{U}(t)=[x, \beta, y, \alpha]^{\mathrm{T}}$ represents the displacement vector of the disk geometric center, the transfer function from the left end of the system to the disk can be expressed as

$$
\left[\begin{array}{cc}
\tilde{\mathbf{T}}(1: 2,1: 2) & \tilde{\mathbf{T}}(1: 2,5: 6) \\
\tilde{\mathbf{T}}(5: 6,1: 2) & \tilde{\mathbf{T}}(5: 6,5: 6)
\end{array}\right]\left[\begin{array}{c}
x \\
\beta \\
y \\
\alpha
\end{array}\right]_{L}+\left[\begin{array}{c}
\Delta M_{x} \\
\Delta Q_{x} \\
\Delta M_{y} \\
\Delta Q_{y}
\end{array}\right]=\left[\begin{array}{c}
x \\
\beta \\
y \\
\alpha
\end{array}\right]_{d}
$$

with $\tilde{\mathbf{T}}=\mathbf{T}_{s 2} \mathbf{T}_{b 2} \mathbf{T}_{s 1} \mathbf{T}_{b 1} \mathbf{T}_{v}$. Combine Eqs. (8)-(9) and rearrange it according to the differential order, the following motion equations can be obtained

$$
\mathbf{M} \ddot{\mathbf{U}}(t)+(\mathbf{D}+\mathbf{G}(t)) \dot{\mathbf{U}}(t)+\mathbf{K}(t) \mathbf{U}(t)=\mathbf{F}(t)
$$

where $\mathbf{M}, \mathbf{D}, \mathbf{G}(t)$ and $\mathbf{K}(t)$ are the mass, damping, gyroscopic and stiffness matrices of the rotating system, respectively. A dot over the displacement vector represents the derivation with time. Notation $\mathbf{F}(t)$ is the force on the system, which can be given as $\mathbf{F}(t)=\left[F_{x}, 0, F_{y}, 0\right]^{\mathrm{T}}$. As the mass of the rotating shaft is included, the general equations of motion of the system will be time-variant. The deterministic ordinary differential equations can be dealt with using the precise integration method or the numerical time integration methods $[48,49]$. 


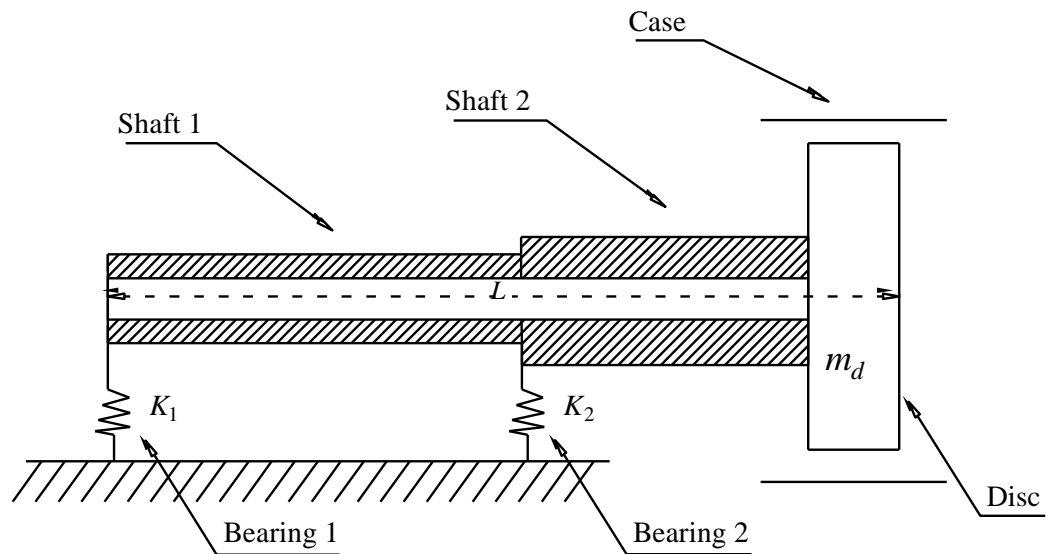

142

Fig. 1. Academic model of the overhung rotor system.

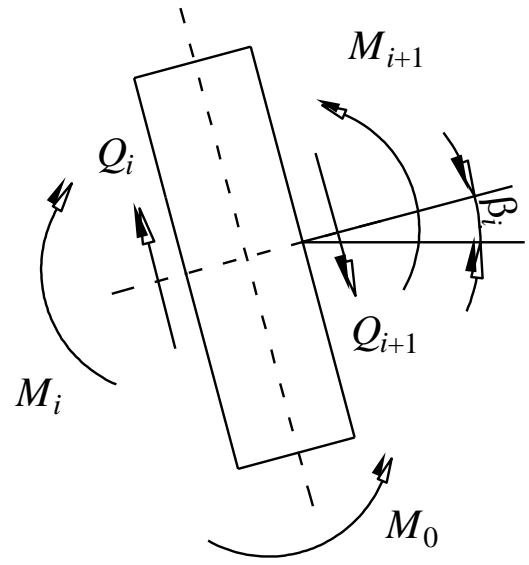

Fig. 2. Transfer mode for rigid disk element.

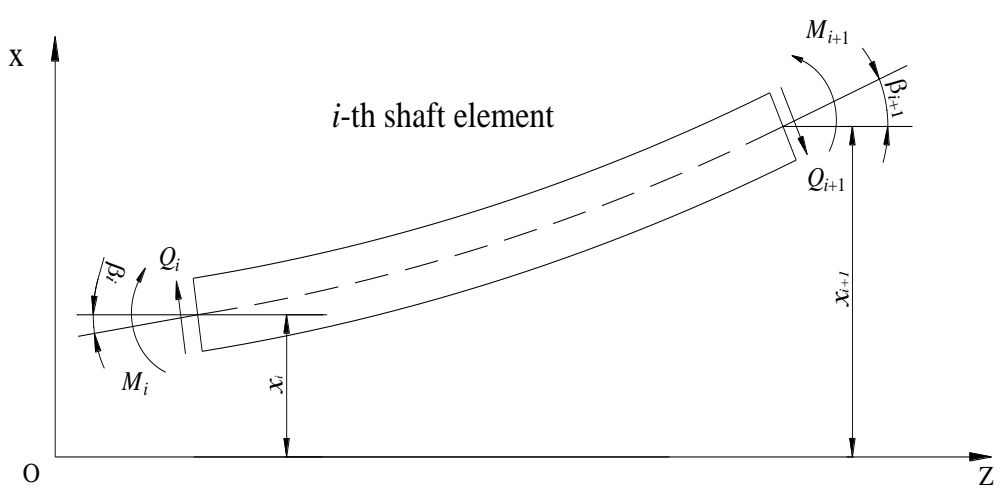

Fig. 3. Transfer mode for shaft element.

\section{Uncertainty representation and quantification}

154 In this section, the representation of different types of parametric uncertainties will be described firstly. 
The underlying uncertain transient dynamic problem is then explained. For the random uncertainties, the non-intrusive PCE is used to quantify their effects based on the Gaussian law. The non-intrusive Chebyshev surrogate is constructed to analyze interval uncertainties. Therefore, the final results will possess the characteristics of both the upper and lower bounds derived from the interval procedure as well as the expectation and variance from the stochastic calculation.

\subsection{Representation of uncertainties and uncertain problem}

As stated previously, the uncertainties are modeled as random and interval variables according to their available prior information. An assumption that all the uncertain parameters are independent is made in this study. With sufficient samples or verified distributions, the uncertainties can be more accurately described as random quantities with precise PDFs. For those inputs, we define a random parameter vector $\mathbf{a}=\left[a_{1}, a_{2}, \cdots, a_{m}\right], m$ denotes the number of random variables. The commonly used Gaussian distribution is adopted here for explicit formulation. For convenience, it can be rewritten as

$$
\mathbf{a}=\overline{\mathbf{a}}\left(1+\delta_{\mathbf{a}} \xi\right)
$$

where $\overline{\mathbf{a}}$ is the mean vector of $\mathbf{a}, \boldsymbol{\delta}_{\mathbf{a}}$ is the standard deviation coefficient vector of $\mathbf{a}$ and $\xi=\left[\xi_{1}, \xi_{2}, \cdots, \xi_{m}\right]$ represents the $m$-dimensional standard Gaussian variable vector. The multiply operation in Eq. (11) is carried out by components. For each of the standard Gaussian variable, we have

$$
\xi_{i} \sim N(0,1), i=1,2, \cdots, m
$$

Then the expectation and variance vector of $\mathbf{a}$ can be expressed as

$$
\left\{\begin{array}{l}
\boldsymbol{\mu}_{\mathrm{a}}=\mathrm{E}[\mathbf{a}]=\left[\mu_{1}, \mu_{2}, \cdots, \mu_{m}\right] \\
\boldsymbol{\sigma}_{\mathrm{a}}^{2}=\left[\sigma_{1}^{2}, \sigma_{2}^{2}, \cdots, \sigma_{m}^{2}\right]
\end{array}\right.
$$

where

$$
\left\{\begin{array}{l}
\mu_{i}=\mathrm{E}\left[a_{i}\right]=\bar{a}_{i} \\
\sigma_{i}^{2}=\bar{a}_{i}^{2} \delta_{i}^{2}
\end{array}, i=1,2, \cdots, m\right.
$$

where operator $E[\square]$ gives the expectation. Eq. (11) links the actual random parameters with the mathematical standard Gaussian variables, which will be beneficial for further manipulations.

Non-probabilistic interval variables are introduced to model the uncertainties whose precise PDFs are unavailable. This occasion is common where the number of samples is small. Then the lower bound (LB) and upper bound (UB) can be used to characterize such input. Similarly, we can define an interval parameter vector $\mathbf{b}^{\mathrm{I}}=\left[b_{1}^{\mathrm{I}}, b_{2}^{\mathrm{I}}, \cdots, b_{n}^{\mathrm{I}}\right], n$ is the number of interval parameters. The superscript $\mathrm{I}$ denotes an interval character. According to interval arithmetic, the interval vector can be expressed by its mid-point vector and radius vector as

$$
\mathbf{b}^{\mathrm{I}}=\mathbf{b}^{\mathrm{c}}+\zeta_{\mathrm{b}}^{\mathrm{I}} \mathbf{b}^{\mathrm{r}}
$$

where $\zeta_{\mathbf{b}}^{\mathrm{I}}$ is the standard interval variable vector, i.e.

$$
\left\{\begin{array}{l}
\zeta_{\mathbf{b}}^{\mathrm{I}}=\left[\zeta_{1}^{\mathrm{I}}, \zeta_{2}^{\mathrm{I}}, \cdots, \zeta_{n}^{\mathrm{I}}\right] \\
\zeta_{j}^{\mathrm{I}}=[-1,1], j=1,2, \cdots, n
\end{array}\right.
$$

Other notations in Eq. (15) are given by 


$$
\left\{\begin{array}{l}
\mathbf{b}^{\mathrm{c}}=\left(\mathbf{b}^{\mathrm{L}}+\mathbf{b}^{\mathrm{U}}\right) / 2 \\
\mathbf{b}^{\mathrm{r}}=\left(\mathbf{b}^{\mathrm{U}}-\mathbf{b}^{\mathrm{L}}\right) / 2
\end{array}\right.
$$

where $\mathbf{b}^{\mathrm{L}}$ and $\mathbf{b}^{\mathrm{U}}$ are the LB and UB vectors of $\mathbf{b}^{\mathrm{I}}$. The transformation in Eq. (15) also connects the actual interval parameters with the mathematical standard interval variables.

In presence of both the random and interval uncertainties, the governing motion equations should be rewritten as

$$
\mathbf{M}\left(\mathbf{a b} \mathbf{b}^{\mathrm{I}}\right) \ddot{\mathbf{U}}\left(\mathbf{a} \mathbf{b}^{\mathrm{I}}\right)+\tilde{\mathbf{C}}\left(\mathbf{a} \mathbf{b}^{\mathrm{I}}\right) \dot{\mathbf{U}}\left(\mathbf{a} \mathbf{b}^{\mathrm{I}}\right)+\mathbf{K}\left(\mathbf{a b} \mathbf{b}^{\mathrm{I}}\right) \mathbf{U}\left(\mathbf{a} \mathbf{b}^{\mathrm{I}}\right)=\mathbf{F}\left(\mathbf{a} \mathbf{b}^{\mathrm{I}}\right)
$$

where $\mathbf{a} \mathbf{b}^{\mathrm{I}}=\left[\mathbf{a} ; \mathbf{b}^{\mathrm{I}}\right]$ collects all the uncertainties, $\tilde{\mathbf{C}}$ is a combination of the damping and gyroscopic terms, and the notation of time $t$ is dropped for simplicity in expression. Equation (18) cannot be solved directly since it is indeterminate and advanced methodologies need to be incorporated, which will be demonstrated in the next subsections. One can firstly deal with either the stochastic uncertainties or the interval ones. If the stochastic ones are considered at first, then the final results will be the LB and UB of the expectation and variance of the transient response. In the other way, they are the expectation and variance of the LB and UB of the dynamic response. Herein, the random type parameters will be handled first. The output solutions can be expressed as

$$
\left\{\begin{array}{l}
\boldsymbol{\mu}^{\mathrm{I}}=[\min \boldsymbol{\mu}(\mathbf{U}(\mathbf{a}, \mathbf{b})), \max \boldsymbol{\mu}(\mathbf{U}(\mathbf{a}, \mathbf{b}))] \\
\left(\sigma^{2}\right)^{\mathrm{I}}=\left[\min \sigma^{2}(\mathbf{U}(\mathbf{a}, \mathbf{b})), \max \sigma^{2}(\mathbf{U}(\mathbf{a}, \mathbf{b}))\right]
\end{array}, \mathbf{b} \in \mathbf{b}^{\mathrm{I}}\right.
$$

\subsection{Polynomial chaos expansion for stochastic uncertainties}

Stochastic modeling of uncertainties can be performed via the PCE in both non-intrusive and intrusive ways $[25,28]$. Generally, the non-intrusive implementation is applied due to its simplicity in concept. It is based on the Karhunen-Loeve expansion in conjunction with the Galerkin projection. Detailed tutorial can be found in literatures on the topic of stochastic finite element methods [50, 51]. For different stochastic distribution types, there are corresponding optimal orthogonal polynomials. The Hermite polynomials are associated with the Gaussian random quantities, which are used in the current study. When dealing with the random uncertainties, the interval parameter vector is kept constant as its mid-value. According to this theory, the stochastic transient response of the rotating system can be expanded on the mean-square convergent polynomial chaos as

$$
\mathbf{U}\left(\mathbf{a}, \mathbf{b}^{\mathrm{I}}\right)=\sum_{k=0}^{\infty} U_{k}\left(\mathbf{b}^{\mathrm{I}}\right) \Psi_{k}(\boldsymbol{\xi})
$$

where $U_{k}\left(\mathbf{b}^{\mathrm{I}}\right)$ is the unknown expansion coefficient and currently deemed as deterministic. $\Psi_{k}(\boldsymbol{\xi})$ represents a rearrangement of the multi-dimensional orthogonal polynomials related to the Gaussian functions which construct a complete basis in the second-order random variable space [25].

In practical computation, the expansion of Eq. (20) can only be accomplished in finite terms. It is truncated after $P$ terms which is the total number of homogenous chaos used and can be given by

$$
P=\frac{(m+p) !}{m ! p !}-1
$$

where $m$ and $p$ are the number of random variables and the order of polynomial chaos, respectively. 
The PCE coefficients is evaluated by

$$
U_{k}\left(\mathbf{b}^{\mathrm{I}}\right)=\frac{E\left[U\left(\mathbf{b}^{\mathrm{I}}, \boldsymbol{\xi}\right) \cdot \Psi_{k}(\boldsymbol{\xi})\right]}{E\left[\Psi_{k}^{2}(\boldsymbol{\xi})\right]}
$$

in which $E\left[\Psi_{k}^{2}(\xi)\right]$ can be calculated based on the orthogonality of the Hermite polynomials

$$
\int_{-\infty}^{\infty} \rho(\xi) \Psi_{i}(\xi) \Psi_{j}(\xi) d \xi=\delta_{i j} E\left[\Psi_{i}^{2}(\xi)\right]
$$

where $\delta_{i j}$ is the Kronecker delta function and $\rho(\xi)=\mathrm{e}^{-\xi^{2} / 2}$ is the weight function associated with the Gaussian distribution. Explicit expressions of $\Psi_{i}^{2}(\xi)$ and $E\left[\Psi_{i}^{2}(\xi)\right]$ can be found in [50].

The remaining crucial step now is to calculate the $E\left[U\left(\mathbf{b}^{\mathrm{I}}, \boldsymbol{\xi}\right) \cdot \Psi_{k}(\boldsymbol{\xi})\right]$. In single-dimensional form, it is expressed as

$$
E\left[U\left(\mathbf{b}^{\mathrm{I}}, \xi\right) \cdot \Psi_{k}(\xi)\right]=\int_{-\infty}^{\infty} \rho(\xi) U\left(\mathbf{b}^{\mathrm{I}}, \xi\right) \Psi_{k}(\xi) d \xi
$$

Equation (24) can be further calculated by the Gaussian-Hermite quadrature which is a numerical technique for continuous integrations

$$
\int_{-\infty}^{\infty} \rho(\xi) U\left(\mathbf{b}^{\mathrm{I}}, \xi\right) \Psi_{k}(\xi) d \xi=\frac{1}{\sqrt{2 \pi}} \sum_{i=0}^{\lambda_{1}} A_{i} U\left(\mathbf{b}^{\mathrm{I}}, \xi_{i}\right) \Psi_{k}\left(\xi_{i}\right)
$$

where $\left\{\xi_{i}\right\}$ are the quadrature points and $\lambda_{1}$ denotes the number of them. $\Psi_{k}\left(\xi_{i}\right)$ and $U\left(\mathbf{b}^{\mathrm{I}}, \xi_{i}\right)$ are the Hermite values and the system response evaluated at $\xi_{i}$, respectively. $A_{i}$ is the quadrature coefficient. Generally, the quadrature points are drawn as the zeros of Hermite polynomials whose order are higher than the chosen PCE order $p$. We use the $p+1$ order term

$$
H(\xi)=(-1)^{p+1} \frac{1}{\sqrt{2 \pi}} e^{\frac{\xi^{2}}{2}}\left[\frac{\mathrm{d}^{p+1}}{\mathrm{~d} \xi^{p+1}} e^{\frac{\xi^{2}}{2}}\right]
$$

The quadrature coefficient in Eq. (25) is given by

$$
A_{i}=\sqrt{\pi} \frac{2^{\lambda_{1}+2}\left(\lambda_{1}+1\right) !}{\left[H_{\lambda_{1}+1}^{\prime}\left(\xi_{i}\right)^{2}\right]}
$$

In multi-dimensional form, the above numerical integration is calculated in tensor form. Generally, the low-order PCE can provide satisfactory estimations. In problems where high order expansion is required, the sparse grid technique or least square regression can be applied to reduce computational burden [42]. It can be observed that the original rotating system model will only be called at quadrature points in the construction of the PCE. In implementation, the Gaussian distribution is truncated and the random samples that have negative values should be removed to guarantee that the physical parameters of the rotor system stay strictly positive [25]. The stochastic transient response of the rotor system considering random uncertainties can then be characterized by the first two statistical moments, i.e. the ensemble mean and the variance

$$
\left\{\begin{array}{l}
\boldsymbol{\mu}\left(\mathbf{U}\left(\mathbf{a}, \mathbf{b}^{\mathrm{I}}\right)\right)=\mathrm{E}\left[\mathbf{U}\left(\mathbf{a}, \mathbf{b}^{\mathrm{I}}\right)\right]=\mathbf{U}_{0}\left(\mathbf{b}^{\mathrm{I}}\right) \\
\boldsymbol{\sigma}^{2}\left(\mathbf{U}\left(\mathbf{a}, \mathbf{b}^{\mathrm{I}}\right)\right)=\sum_{i=1}^{P} \mathbf{U}_{i}^{2}\left(\mathbf{b}^{\mathrm{I}}\right) E\left[\Psi_{i}^{2}\left(\boldsymbol{\xi}_{i}\right)\right]
\end{array}\right.
$$

\subsection{Chebyshev surrogate method for interval uncertainties}

In the last subsection, random uncertainties are modeled and the mean and variance of the stochastic 
response have been produced. Now, we have to take the effects of interval parameters into consideration, which leads to the calculation of the interval mean response and interval variance. The main objective is to find the $\min / \max$ values of the first two statistical moments for each element. Direct interval arithmetic or the intrusive interval methods have their disadvantages with respect to overestimation control and application convenience in engineering problems. Alternatively, we use the non-intrusive Chebyshev surrogate [32] to model the uncertain problem regarding the procedure of the PCE and the original rotor system as a black box. Based on Eqs. (15) and (28), the practical interval parameters have been transformed to a set of standard interval variables $\zeta_{\mathrm{b}}^{\mathrm{I}}$ and we can model with the latter equivalently. Their projection relationship can be expressed as

$$
\zeta_{\mathbf{b}}^{\mathrm{I}}=\frac{\mathbf{b}^{\mathrm{I}}-\mathbf{b}^{\mathrm{c}}}{\mathbf{b}^{\mathrm{r}}}
$$

where calculation is done in components. Let $\mathbf{Y}=[\mathbf{Y} 1, \mathbf{Y} 2]=\left[\boldsymbol{\mu}\left(\mathbf{b}^{\mathrm{I}}\right), \sigma^{2}\left(\mathbf{b}^{\mathrm{I}}\right)\right]$, the goal is to determine the bound values for every element in the $\mathbf{Y}\left(\mathbf{b}^{\mathrm{I}}\right)$. By using Eq. (29) and the $q$-order Chebyshev approximation, the following surrogate can be established

$$
\mathbf{Y}(\zeta)=\sum_{i_{1}=0}^{q} \cdots \sum_{i_{n}=0}^{q} \frac{1}{2^{v}} \eta_{i_{1} i_{2} \cdots i_{n}} C_{i_{1} i_{2} \ldots i_{n}}(\zeta), \zeta \in[-1,1]^{n}
$$

where $\eta_{i_{1} i_{2} \ldots i_{n}}$ is the Chebyshev expansion coefficient and $v$ represents the appearance times of zero in the subscript indices $i_{1}, i_{2} \cdots, i_{n} . C_{i_{1} i_{2} \cdots i_{n}}$ denotes the $n$-dimensional Chebyshev polynomial

$$
C_{i_{1} i_{2} \cdots i_{n}}(\zeta)=C_{i_{1}}\left(\zeta_{1}\right) C_{i_{2}}\left(\zeta_{2}\right) \cdots C_{i_{n}}\left(\zeta_{n}\right)
$$

where the single-dimensional Chebyshev polynomial is expressed as

$$
\left.C_{i}(\zeta)=\cos (i \arccos \zeta)\right), \quad \zeta \in[-1,1]
$$

In recurrence form, it is given by

$$
\left\{\begin{array}{l}
C_{0}(\zeta)=1, C_{1}(\zeta)=\zeta \\
C_{k+1}(\zeta)=2 \zeta C_{k}(\zeta)-C_{k-1}(\zeta)
\end{array}, \zeta \in[-1,1]\right.
$$

Its zeros are often used as numerical integration points, which are calculated by

$$
\zeta^{(k)}=\frac{(2 k-1) \pi}{2 q}, k=1,2, \cdots, \lambda_{2}
$$

where $\lambda_{2}$ is the number of integration points used and it is set to $\lambda_{2}=q+1$ in this study. The expansion coefficients can be evaluated by the Gaussian-Chebyshev quadrature in tensor way using all of the Chebyshev points. The number of the interpolation point pool in multi-dimensional cases is $N=\lambda_{2}^{n}=(q+1)^{n}$, which may be unaffordable for large-scale problems. A collocation sampling scheme using fewer samples was proposed in [32]. According to this approach, the coefficients can be obtained alternatively by the regression technique. Delete the terms in Eq. (30) whose order is higher than $q$ and the number of remaining terms is $N^{\prime}=(n+q) ! /(n ! q !)$. It is suggested that $\hat{N}=2 N^{\prime}$ collocations should be kept in order to be robust if $N>2 N^{\prime}$ holds true [52]. Since the number of unknown coefficients does not match the number of the sample points, the least square formulation can be employed to solve the problem as

$$
\boldsymbol{\eta}=\left(\boldsymbol{\Phi}^{\mathrm{T}} \boldsymbol{\Phi}\right)^{-1} \boldsymbol{\Phi}^{\mathrm{T}} \tilde{\mathbf{Y}}(\tilde{\boldsymbol{\zeta}})
$$

where $\tilde{\zeta}$ denotes the $\hat{N} \times n$ sample matrix. $\tilde{\mathbf{Y}}(\tilde{\zeta})$, which is deterministic, represents the ensemble 
mean and variance output matrix calculated via the PCE procedure at those sample points. The transformation matrix $\Phi$ is composed of the values of the multi-dimensional Chebyshev polynomials evaluated at sample points and it is given in elements as

$$
\boldsymbol{\Phi}=\left[\begin{array}{ccccc}
C_{0, \cdots, 0}\left(\tilde{\zeta}_{1}\right) & \ldots & C_{i_{1}, \cdots, i_{n}}\left(\tilde{\boldsymbol{\zeta}}_{1}\right) & \ldots & C_{0, \cdots, q}\left(\tilde{\boldsymbol{\zeta}}_{1}\right) \\
C_{0, \cdots, 0}\left(\tilde{\zeta}_{2}\right) & \ldots & C_{i_{1}, \cdots, i_{n}}\left(\tilde{\boldsymbol{\zeta}}_{2}\right) & \ldots & C_{0, \cdots, q}\left(\tilde{\boldsymbol{\zeta}}_{2}\right) \\
\vdots & \ddots & \vdots & \ddots & \vdots \\
C_{0, \cdots, 0}\left(\tilde{\boldsymbol{\zeta}}_{\hat{N}}\right) & \cdots & C_{i_{1}, \cdots, i_{n}}\left(\tilde{\boldsymbol{\zeta}}_{\hat{N}}\right) & \ldots & C_{0, \cdots, q}\left(\tilde{\boldsymbol{\zeta}}_{\hat{N}}\right)
\end{array}\right]_{\hat{N} \times N^{\prime}}
$$

where $i_{1}+\cdots+i_{n} \in[0, q]$. The expression for statistical moments output matrix of the system is

$$
\tilde{\mathbf{Y}}(\tilde{\boldsymbol{\zeta}})=\left[\begin{array}{cc}
\boldsymbol{\mu}\left(\mathbf{a}, \tilde{\zeta}_{1}\right) & \boldsymbol{\sigma}^{2}\left(\mathbf{a}, \tilde{\zeta}_{1}\right) \\
\vdots & \vdots \\
\boldsymbol{\mu}\left(\mathbf{a}, \tilde{\zeta}_{\hat{N}}\right) & \boldsymbol{\mu}\left(\mathbf{a}, \tilde{\zeta}_{\hat{N}}\right)
\end{array}\right]
$$

When the unknown coefficients of Eq. (30) are solved, the surrogate model is fully determined. Optimization or the scanning method can be incorporated to search the $\min / \max$ values of the simplex surrogate function without much effort. The complete set of results to characterize the transient response of the rotating system considering both random and interval uncertainties are: the upper bounds of the expectation (UBE) and variance (UBV), and the lower bounds of the expectation (LBE) and variance (LBV). Based on Eqs. (19) and (28), they are expressed as

$$
\begin{gathered}
\boldsymbol{\mu}^{\mathrm{I}}=\left[\boldsymbol{\mu}^{\mathrm{L}}, \boldsymbol{\mu}^{\mathrm{U}}\right]=\left[\underset{\zeta \in[-1,1]^{n}}{\min }(\mathbf{Y} 1), \max _{\zeta \in[-1,1]^{n}}(\mathbf{Y} 1)\right] \\
\left(\boldsymbol{\sigma}^{2}\right)^{\mathrm{I}}=\left[\left(\boldsymbol{\sigma}^{2}\right)^{\mathrm{L}},\left(\boldsymbol{\sigma}^{2}\right)^{\mathrm{U}}\right]=\left[\min _{\zeta \in[-1,1]^{n}}(\mathbf{Y}), \max _{\zeta \in[-1,1]^{n}}(\mathbf{Y} 2)\right]
\end{gathered}
$$

\section{Numerical results and discussion}

In this section, we will investigate several cases with different random and interval uncertainties. The values of the deterministic physical parameters of the rotor are given in Table 1. To provide a clear outline of the simulation results, the parameter sets are summarized in Table 2 for each case. It should be noted that the hybrid procedure developed can be applied to problems with only random or interval uncertainties. The variability of the responses will demonstrate the sensitivity of the uncertain parameter and one can choose to prioritize those with higher impacts in design and optimizations. For better comprehension in the hybrid cases, we present the results with either random parameter or interval parameter firstly which correspond to Case 1 and 2. All the transient responses are provided as the deflection of the geometric center of disk, i.e. $\sqrt{x^{2}+y^{2}}$. Figure 4 plots the stochastic response of the rotating system characterized by the expectation and standard deviation considering random viscous damping using the PC order 3, where the standard deviation is the square root of the variance. To demonstrate the effects of the deviation of random damping on the stochastic output, three conditions $(5 \%, 10 \%$ and $15 \%)$ are considered for simulations and the corresponding standard deviations of the response are shown in Fig. 5 as supplementary results for Case 1. The mean of the response is unchanged and it is omitted in Fig. 5. It can be observed that larger deviation in the random damping leads greater variability in the response. The interval responses are demonstrated in Fig. 6 using the 
319 Chebyshev order 3 when the eccentricity of the unbalance is treated as bounded uncertainty. From Figs.

3204 and 6, we can observe that the two kinds of uncertainties will lead to different outputs of the response.

321 The expectation of the transient response is identical to the deterministic solution in this case, which is 322 naturally and essential. Along with the response expectation, a standard deviation curve is presented to

323 denote the deviation ability of the response. The deviation here mainly denotes the influence of the 324 random uncertainty on the responses. In Fig. 4, fierce fluctuations in the attenuation zone of the standard 325 deviation can be noticed, which is influenced by the uncertainty in the viscous damping. The response 326 range demonstrated in Fig. 6 exhibits the influence of the mass unbalance and the nominal curve is 327 perfectly enclosed in the bounds. A preliminary impression is provided through these results and the 328 uncertain responses with hybrid uncertainties can be better comprehended. The accuracy of the hybrid 329 procedure will be validated in the following cases with multiple uncertainties and therefore this two 330 cases will not be verified to maintain concise.

Table 1 Value of physical parameters of the rotor.

\begin{tabular}{lll}
\hline Symbol & Description & Value \\
\hline$E$ & Young's modulus of elasticity & $2.1 \times 10^{11} \mathrm{~N} / \mathrm{m}^{2}$ \\
$L_{1}$ & Length of shaft 1 & $0.3 \mathrm{~m}$ \\
$L_{2}$ & Length of shaft 2 & $0.12 \mathrm{~m}$ \\
$D_{1}$ & Outer diameter of shaft 1 & $0.01 \mathrm{~m}$ \\
$D_{2}$ & Outer diameter of shaft 2 & $0.02 \mathrm{~m}$ \\
$D_{0}$ & Inner diameter of shafts & $0.03 \mathrm{~m}$ \\
$K_{1}$ & Stiffness of support 1 & $1 \times 10^{8} \mathrm{~N} / \mathrm{m}$ \\
$K_{2}$ & Stiffness of support 2 & $1 \times 10^{6} \mathrm{~N} / \mathrm{m}$ \\
$C$ & Viscous damping & $120 \mathrm{~N} \cdot \mathrm{s} / \mathrm{m}$ \\
$m$ & Disk mass & $8.4 \mathrm{Kg}$ \\
$e$ & Eccentricity & $8 \times 10^{-5} \mathrm{~m}$ \\
$\rho$ & Density & $7800 \mathrm{Kg} / \mathrm{m}^{3}$ \\
$I_{p}$ & Polar mass moment of inertia & $0.0695 \mathrm{Kg} \cdot \mathrm{m}^{2}$ \\
$I_{d}$ & Transverse mass moment of inertia & $0.0357 \mathrm{Kg} \cdot \mathrm{m}^{2}$ \\
\hline
\end{tabular}

Table 2 Parameter sets for simulations.

\begin{tabular}{lllllll}
\hline Case & $e / \mathrm{m}$ & $K_{1} /(\mathrm{N} / \mathrm{m})$ & $K_{2} /(\mathrm{N} / \mathrm{m})$ & $C /(\mathrm{N} \cdot \mathrm{s} / \mathrm{m})$ & $\rho /\left(\mathrm{kg} / \mathrm{m}^{3}\right)$ & $E /\left(\mathrm{N} / \mathrm{m}^{2}\right)$ \\
\hline 1 & $8 \times 10^{-5}$ & $1 \times 10^{8}$ & $1 \times 10^{6}$ & $N\left(120,12^{2}\right)$ & 7800 & $2.1 \times 10^{11}$ \\
2 & {$[7.2,8.8] \times 10^{-5}$} & $1 \times 10^{8}$ & $1 \times 10^{6}$ & 120 & 7800 & $2.1 \times 10^{11}$ \\
3 & {$[7.2,8.8] \times 10^{-5}$} & $1 \times 10^{8}$ & $1 \times 10^{6}$ & $N\left(120,6^{2}\right)$ & 7800 & $2.1 \times 10^{11}$ \\
4 & {$[7.2,8.8] \times 10^{-5}$} & $1 \times 10^{8}$ & {$[0.95,1.05] \times 10^{6}$} & $N\left(120,6^{2}\right)$ & 7800 & $N\left(2.1 \times 10^{11},\left(2.1 \times 10^{9}\right)^{2}\right)$ \\
5 & {$[6.8,9.2] \times 10^{-5}$} & {$[0.9,1.1] \times 10^{8}$} & {$[0.9,1.1] \times 10^{6}$} & $N\left(120,12^{2}\right)$ & $N\left(7800,156^{2}\right)$ & $N\left(2.1 \times 10^{11},\left(6.3 \times 10^{9}\right)^{2}\right)$ \\
\hline
\end{tabular}



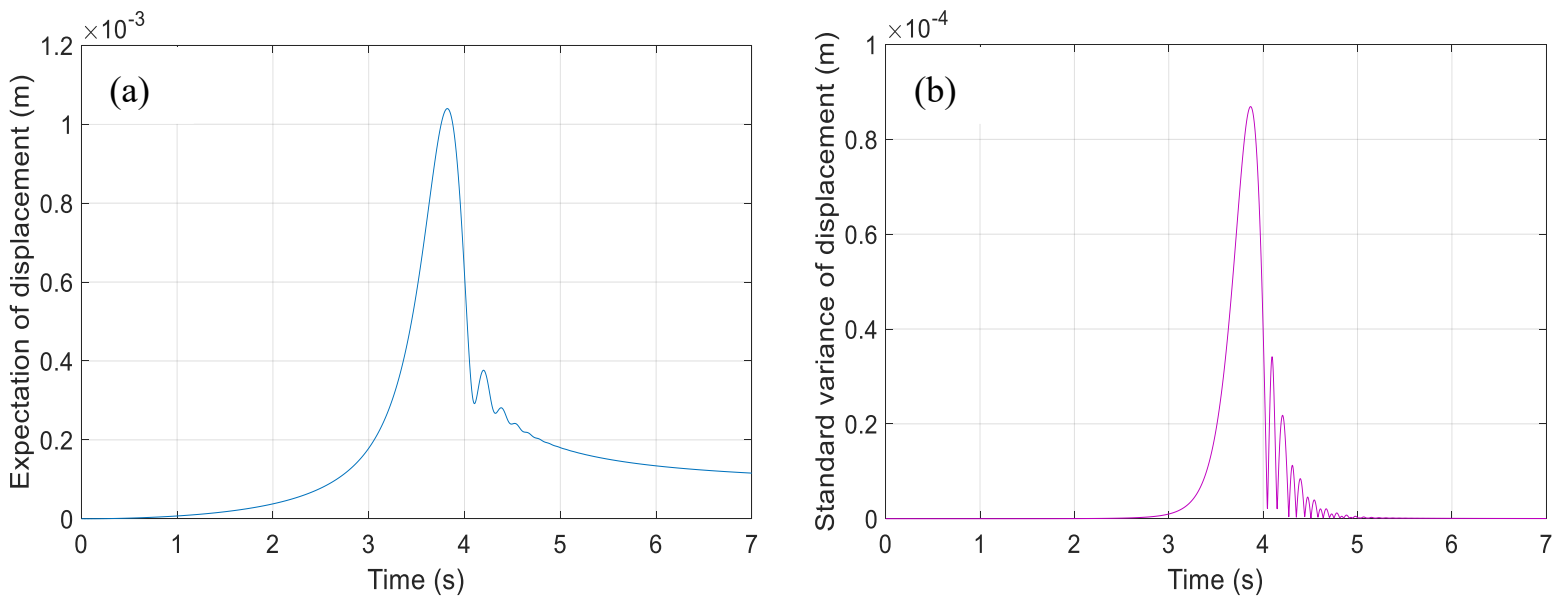

Fig. 4. Stochastic transient response of the rotor with random viscous damping (Case 1): (a) expectation and (b) standard deviation.

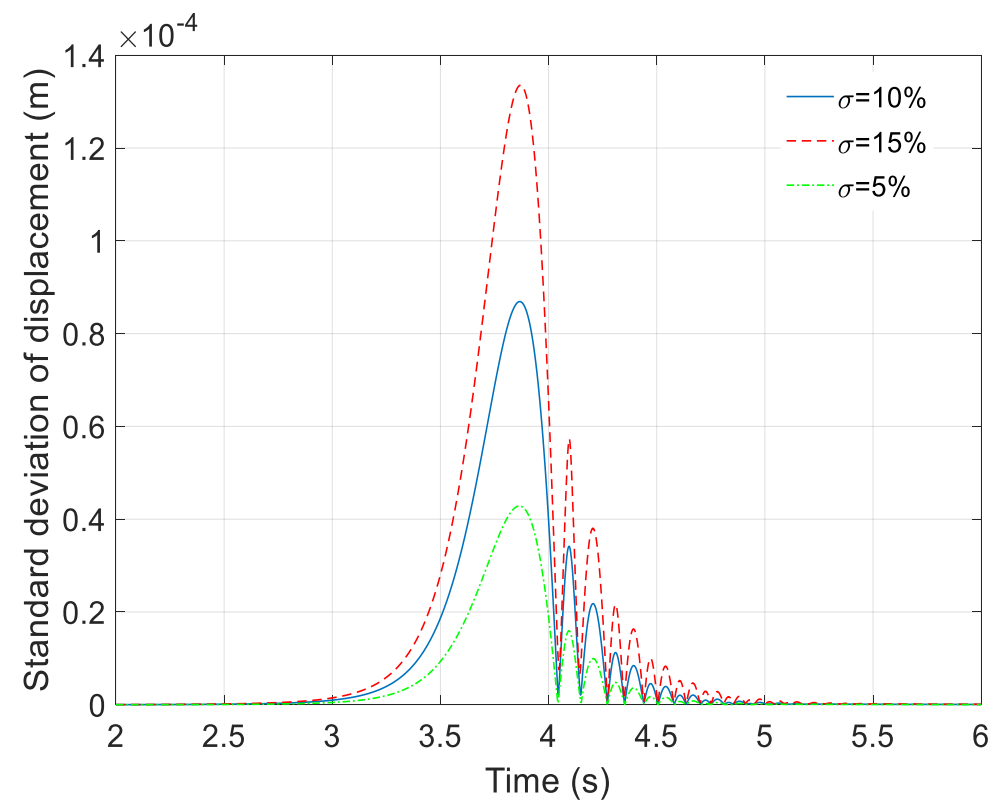

In hybrid problems, we study Case 3 first where the eccentricity is taken as an interval parameter and the viscous damping is considered to be a random variable. This is a single random-single interval problem and the deviation ability of them is $10 \%$ of their nominal values. In this case, the output will possess the characteristics of both the probabilistic and non-probabilistic quantities. To verify the accuracy of the results, the classic sampling methods are applied to provide reference solutions. The MCS will be used to draw random samples. The scanning method is employed for the interval parameter. Suppose $\kappa_{1}$ evenly distributed samples are used to deal with the interval uncertainty and $\kappa_{2}$ samples are drawn for the random uncertainty, the total sample points needed to obtain the reference solution will be $\kappa=\kappa_{1} \times \kappa_{2}$ in this case. At every sample point for the interval parameter, a pair of expectation and variance of the response will be produced 


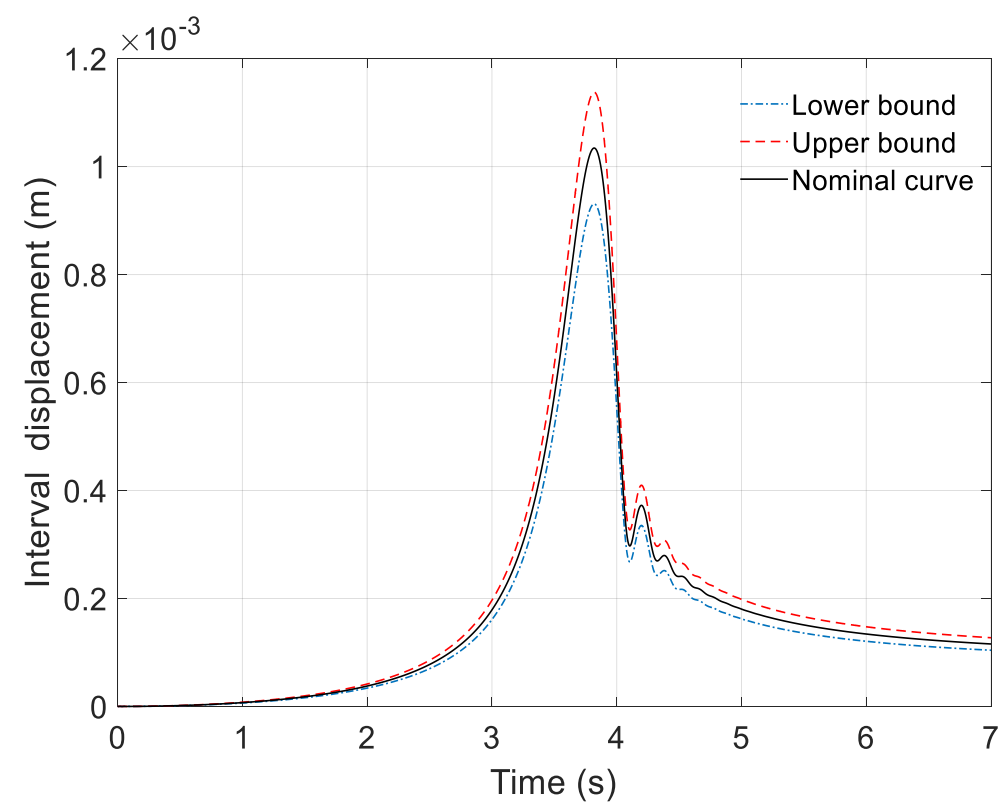

Fig. 6. Interval transient response of the rotor with bounded unbalance eccentricity (Case 2).

$$
\left\{\begin{array}{l}
\tilde{\boldsymbol{\mu}}_{j}=\frac{1}{\kappa_{2}} \sum_{i=1}^{\kappa_{2}} \hat{\mathbf{U}}_{i j}\left(\tilde{\mathbf{a}}_{i}, \tilde{\mathbf{b}}_{j}\right) \\
\tilde{\boldsymbol{\sigma}}_{j}^{2}=\frac{1}{\kappa_{2}-1} \sum_{i=1}^{\kappa_{2}}\left(\hat{\mathbf{U}}_{i j}\left(\tilde{\mathbf{a}}_{i}, \tilde{\mathbf{b}}_{j}\right)-\boldsymbol{\mu}_{j}\right)^{2}
\end{array}, j=1,2, \cdots, \kappa_{1}\right.
$$

where $\tilde{\mathbf{a}}_{i}$ and $\tilde{\mathbf{b}}_{j}$ are the samples in tensor grid for the interval and random uncertainties. The $\hat{\mathbf{U}}_{i j}$ represents the deterministic response at these sample points, which can be expressed in matrix as

$$
[\tilde{\mathbf{a}}, \tilde{\mathbf{b}}]=\left[\begin{array}{ccc}
\left(\xi_{1}, \zeta_{1}\right) & \cdots & \left(\xi_{1}, \zeta_{\kappa_{2}}\right) \\
\vdots & \ddots & \vdots \\
\left(\xi_{\kappa_{1}}, \zeta_{1}\right) & \cdots & \left(\boldsymbol{\xi}_{\kappa_{1}}, \zeta_{\kappa_{2}}\right)
\end{array}\right]_{\kappa_{1} \times \kappa_{2}}
$$

After cycle for all the samples, the bounds of them can be obtained. It is expressed as

$$
\left\{\begin{array}{l}
\boldsymbol{\mu}_{r e f}^{\mathrm{I}}=[\min \{\tilde{\boldsymbol{\mu}}\}, \max \{\tilde{\boldsymbol{\mu}}\}] \\
\boldsymbol{\sigma}_{\text {ref }}^{\mathrm{I}}=[\min \{\tilde{\boldsymbol{\sigma}}\}, \max \{\tilde{\boldsymbol{\sigma}}\}]
\end{array}\right.
$$

It should be noted that this process will be time-consuming since the total run times of the rotor analysis model will be large. For Case 3, we used 20 scanning points and 1000 random samples to calculate the reference results, which means the deterministic problem will run 20000 times. Figure 7 presents the interval expectation and interval standard deviation of the uncertain response of the rotor calculated using the proposed procedure as well as the reference solutions. As illustrated in Fig. 7, the results from the two schemes are in good agreement, including the attenuation areas where the standard deviation is subject to intense fluctuations. For an insightful comparison, a magnified local view of the standard deviation shown in Fig. 7(b) is provided in Fig. 8. It is worth mentioning that only order 3 for both the PCE and Chebyshev approximation is used. It can be seen that the bounds of the expectation obtained from the proposed method accord with the reference solution ranges. The standard deviation bounds of the response from the hybrid procedure enclose the reference solution range and only trivial differences 

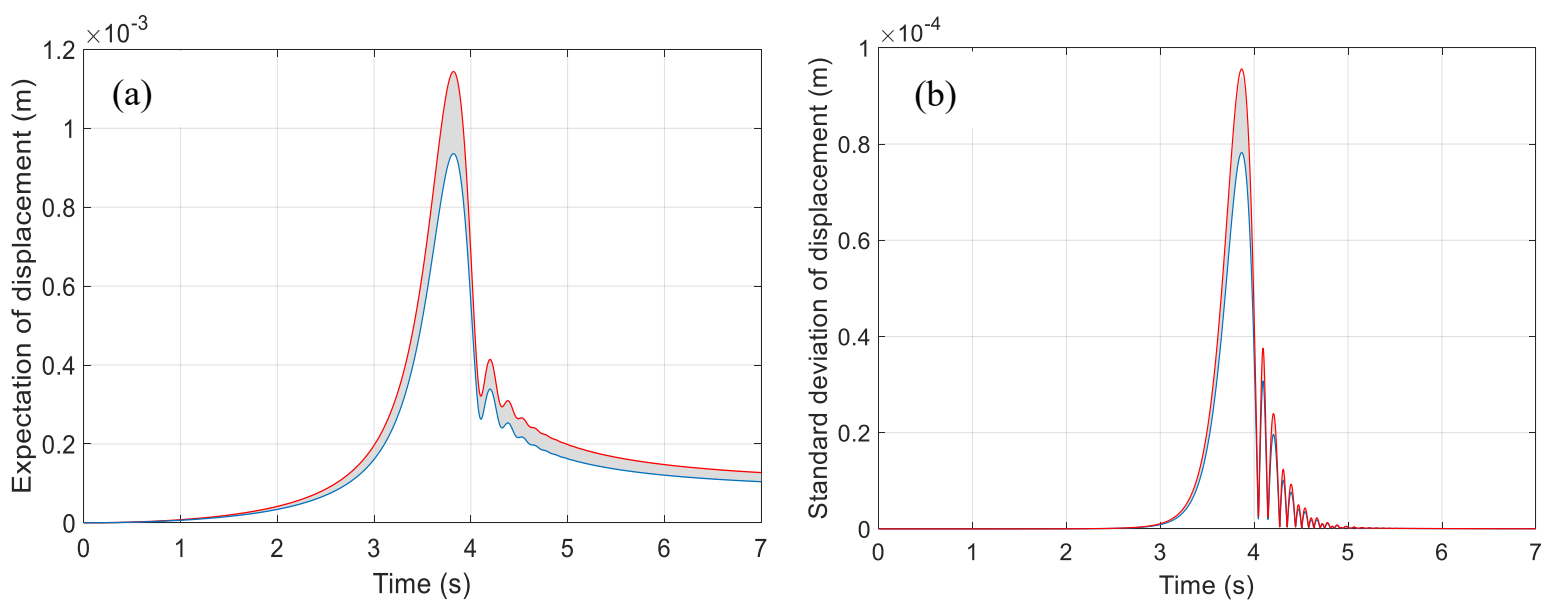

Fig. 7. Transient response of the rotor with interval unbalance and random viscous damping (Case 3): (solid linesresponse bounds; grey area-reference solution ranges) (a) interval expectation and (b) interval standard deviation.

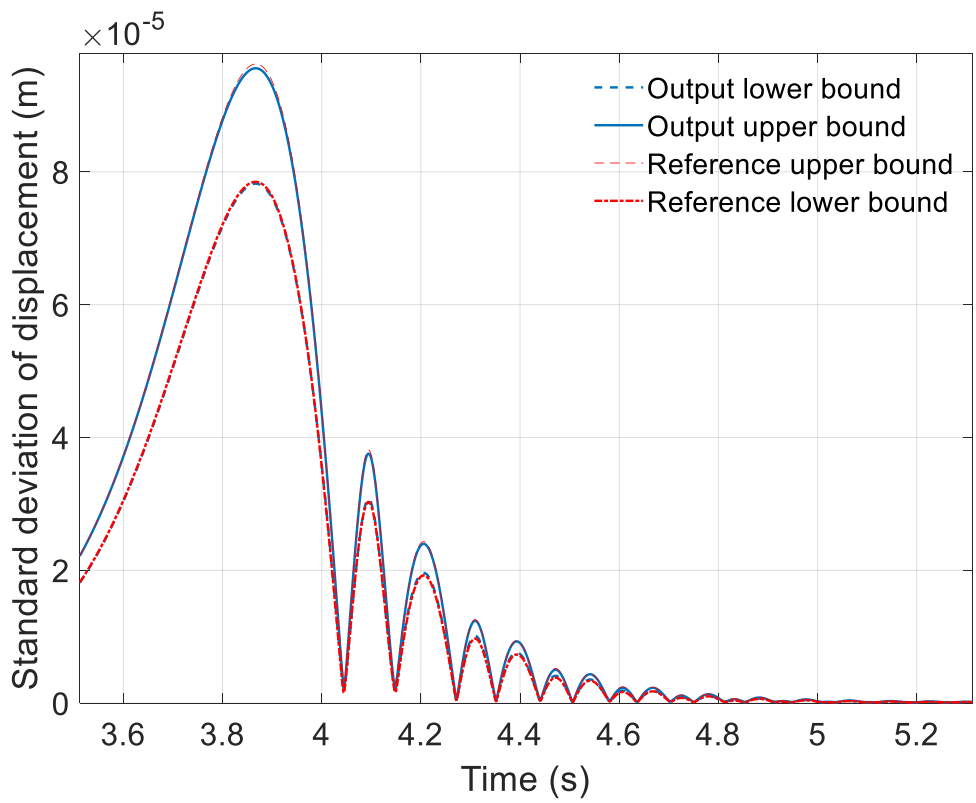

Fig. 8. Local view of the standard deviation comparison between the hybrid procedure and reference solution.

In Case 4, we deal with two interval parameters and two random parameters, i.e. the interval unbalance and interval stiffness of support $K_{2}$, and the random damping and Young's modulus $C, E$. In fact, the stiffness of a support or bearing can be difficult to accurately define or measure and it is often being adjusted to satisfy the design requirements. Many researchers chose to treat the stiffness of bearings as uncertain variables in their investigations [24, 25, 53]. Ma et al. [33] pointed out that the supporting stiffness in a rotor system can be composed of different parts and it is influenced by many factors such as temperature and loads. Then, they considered the stiffness of supports as uncertain parameters. Due to limited prior information in engineering, it is better to describe them as interval quantities. The material properties of the elastic shaft are then modeled as random variables. For this complex problem 
with multiple different kinds of uncertainties, the simulation results are given in Fig. 9 using an order 4 in the PCE and interval method. From Fig. 9, the uncertain response bounds have different features compared with Case 3. The fluctuations in the attenuation zone are less significant and the upper bounds have flat-peak bands $[33,54]$, which is an indication of the influences of uncertainties on the natural frequency of the rotor system. To further demonstrate its effectiveness in dynamic problems with more uncertain parameters, a large number of uncertainties are included for Case 5 where there are three interval quantities and three random uncertainties. The specific uncertain physical parameters considered and the variabilities of these uncertainties can be found in Table 1. Figure 10 shows the first two interval statistical moments of the transient response of the rotor system for Case 5. The results combine the characteristics of the previous cases and illustrate the behaviors of the rotor under large number of uncertain parameters. We can observe from Fig. 10 that the possible ranges for the statistical of moments are wider compared with the former cases. The standard deviation range in Fig. 10(b) suggests that the deviation ability of the response far from the resonance region is very low and its peak is observed also at the resonance point. There are minor oscillations in the lower bounds of the standard deviation in the attenuation area, which can be eliminated by increasing the expansion orders.

It is worth pointing out that the results for the MCS with samples fewer than 1000 are not reliable because of the poor convergence rate. The number of samples for interval parameters also grow in power basis with dimension. Therefore, simulations for Case 4 and 5, which involve large number of uncertainties, may be nearly impossible for the reference sampling methods because of the underlying prohibitive computational demand. The perturbation method will not be applicable here as large range uncertainty is present. In derivative-based methods such as the Taylor method or the Legendre method [55], it is hard to extend to high orders due to the difficulties in derivation or the symbolic computation will be very time-consuming. Moreover, they are all intrusive methods which are difficult to implement.

412 To provide comparisons with other established non-intrusive methods, the CIF [31] coupled with the 413 PCE is utilized as another framework for Case 5 with multiple uncertainties. The CIF is vastly used recently in the UQ of different fields of dynamics and can provide results with acceptable overestimations. Moreover, the PCE is still used for this alternative frame due to its popularity in stochastic computations. The relative differences of the results obtained from the proposed model and the CIF-PCE are plotted in Fig. 11 with respect to the upper and lower bounds of responses. It can be seen that the two solutions are in agreement and only minor discrepancies are noticed as demonstrated in the magnitudes of differences in Fig. 11. Most of differences occurred in the attenuation area where fluctuations are heavy. It should be noted that no strategies aimed at reducing the computation burden are incorporated in the CIF-PCE. Therefore, the CPU time needed for this six-dimensional uncertain problem can be a considerable amount. A desktop with Intel Core i7-4770@3.4GHz and RAM 16.0GB is used for the calculation. The average CPU time elapsed to complete the task is $15308.65 \mathrm{~s}$ for the proposed analysis model and $56938.82 \mathrm{~s}$ for the CIF-PCE. Based on the above comparative analysis, it is clear that the proposed model has obvious advantage in efficiency and has equivalent accuracy as the 

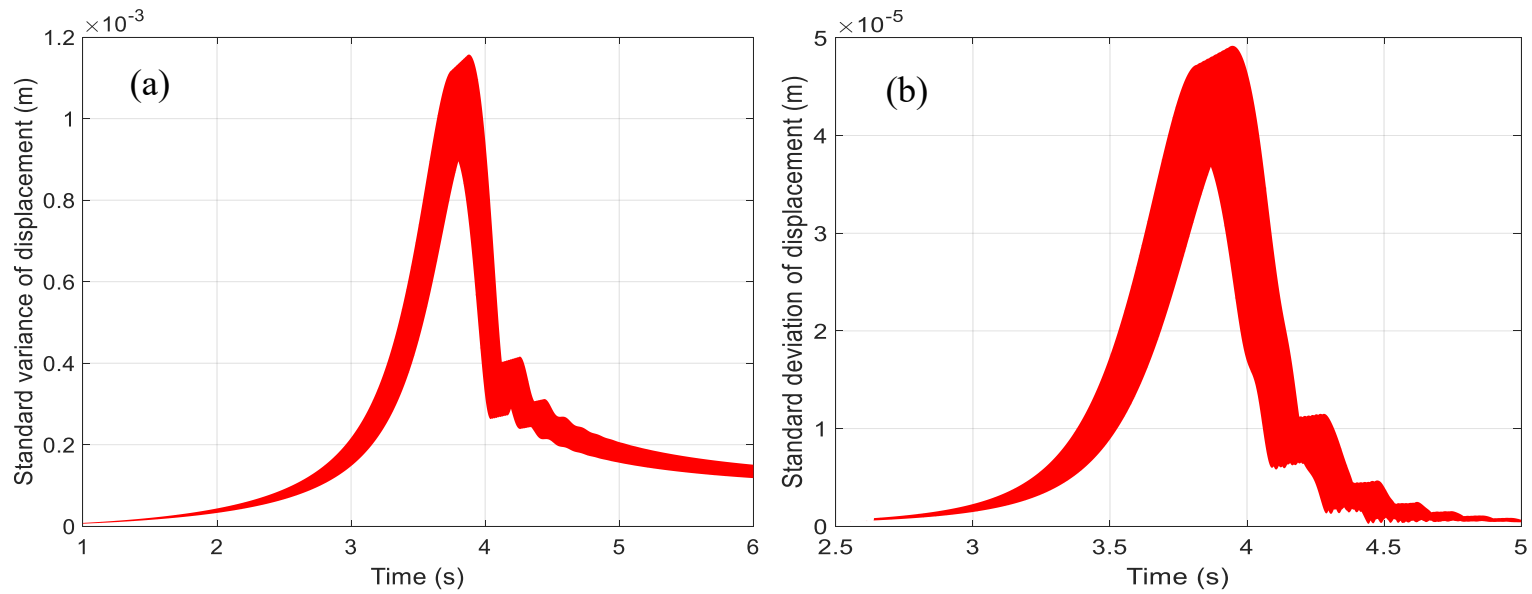

Fig. 9. Transient response of the rotor with two interval uncertainties and two random uncertainties (Case 4): (a)
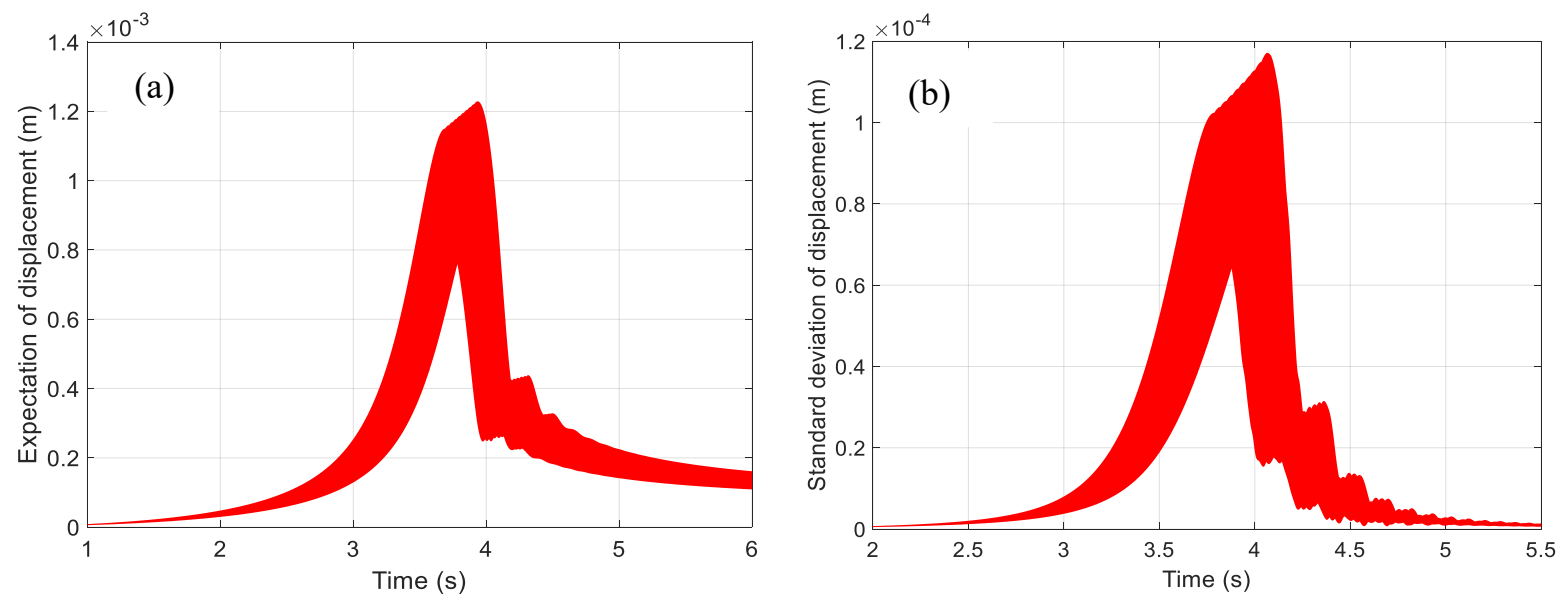

Fig. 10. Transient response of the rotor with three interval uncertainties and three random uncertainties (Case 5):
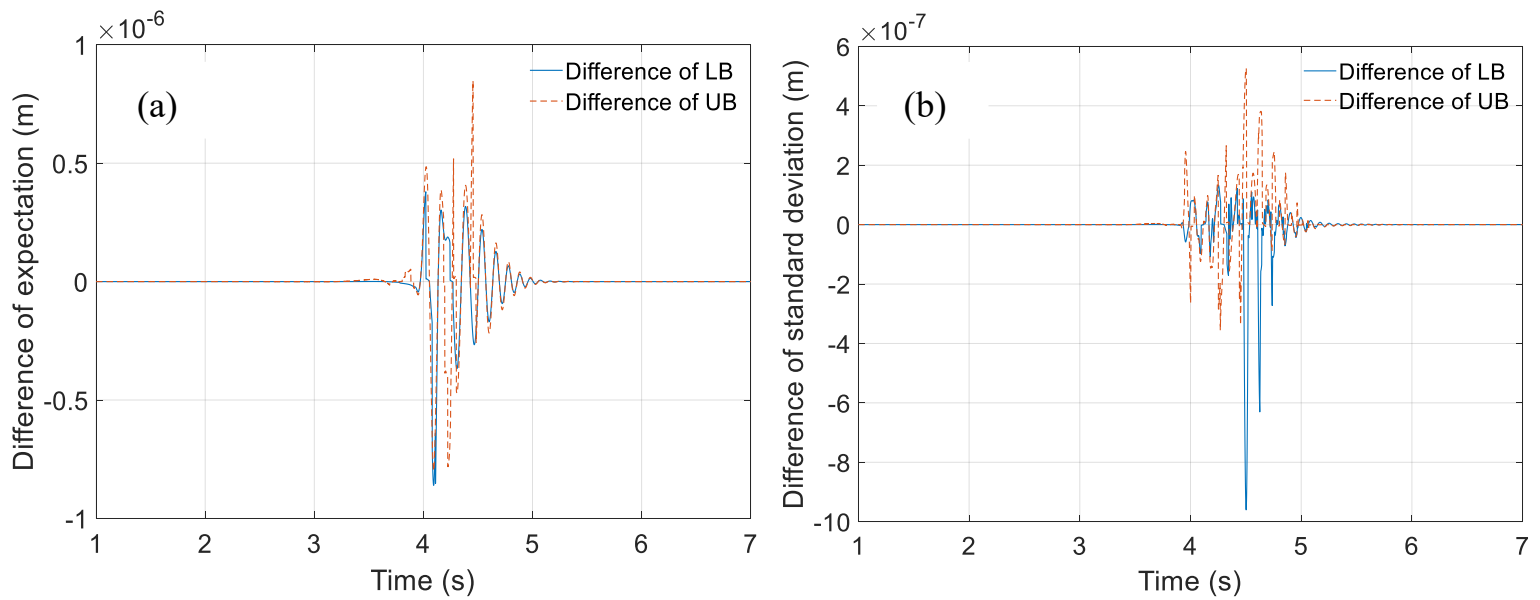

436 Fig. 11. Difference of the results obtained from two hybrid uncertainty analysis methods (Case 5): (a) expectation and (b) standard deviation. 


\section{Conclusions}

440 The interval statistical moments of the transient vibration responses of a hollow overhung rotor system are studied considering both random and interval parameters via a non-intrusive uncertainty propagation scheme, which combines the Polynomial Chaos Expansion and the Chebyshev surrogate method. It has shown its effectiveness in problems with hybrid uncertainties or either type of them. The accuracy of the method are demonstrated by the reference solutions derived from the Monte Carlo simulation in conjunction with the scanning method and other hybrid procedures. Simulations with a large number of uncertain parameters have been carried out. The numerical results indicate that the transient responses of the rotor is affected by the hybrid parametric uncertainties and it is more reasonable to take them into consideration in the design and dynamic analysis of such systems. In the meantime, response analysis of the individual uncertain parameters will help to evaluate their sensitivities and those with higher ones should be prioritized in design and optimizations. The approach developed in this study can also be adapted to other dynamic problems with hybrid uncertainties conveniently due to the fact that no modifications should be made to the well-established solution process.

\section{Acknowledgements}

This work acknowledges the financial support from the National Natural Science Foundation of China (No. 11272257) and the Fundamental Research Funds for the Central Universities (No. 3102018ZY016).

\section{References}

[1] T.R. Babu, S. Srikanth, A.S. Sekhar, Hilbert-Huang transform for detection and monitoring of crack in a transient rotor, Mech. Syst. Signal Pr., 22 (2008) 905-914.

458 [2] Y. Yang, Y. Yang, D. Cao, G. Chen, Y. Jin, Response evaluation of imbalance-rub-pedestal looseness coupling fault on a geometrically nonlinear rotor system, Mech. Syst. Signal Pr., 118 (2019) 423-442.

460 [3] A.K. Darpe, K. Gupta, A. Chawla, Transient response and breathing behaviour of a cracked Jeffcott rotor, J. Sound Vib., 272 (2004) 207-243. [4] F.K. Choy, J. Padovan, Non-linear transient analysis of rotor-casing rub events, J. Sound Vib., 113 (1987) 529-545.

[5] C. Yue, X. Ren, Y. Yang, W. Deng, Unbalance identification of speed-variant rotary machinery without phase angle measurement, Shock Vib., (2015) Article ID 934231.

466 [6] L. Nechak, J.J. Sinou, Hybrid surrogate model for the prediction of uncertain friction-induced instabilities, 467 J. Sound Vib., 396 (2017) 122-143.

468 [7] P.M. Pawar, R. Ganguli, Genetic fuzzy system for online structural health monitoring of composite helicopter rotor blades, Mech. Syst. Signal Pr., 21 (2007) 2212-2236.

470 [8] I. Elishakoff, D. Livshits, Some closed-form solutions in random vibration of Bernoulli-Euler beams. Int. 471 J. Eng. Sci., 22 (1984) 1291-1301.

472 [9] T. Roncen, J.J. Sinou, J.P. Lambelin, Experiments and nonlinear simulations of a rubber isolator subjected 473 to harmonic and random vibrations, J. Sound Vib., 451 (2019) 71-83.

474 [10] J.J. Sinou, L. Nechak, S. Besset, Kriging Metamodeling in Rotordynamics: Application for predicting critical speeds and vibrations of a flexible rotor, Complexity, (2018) 1264619.

476 [11] Y. Yang, Q. Wu, Y. Wang, W. Qin, K. Lu, Dynamic characteristics of cracked uncertain hollow-shaft, 
Mech. Syst. Signal Pr., 124 (2019) 36-48.

478 [12] G. Muscolino, R. Santoro, Dynamics of multiple cracked prismatic beams with uncertain-but-bounded 479 depths under deterministic and stochastic loads, J. Sound Vib., 443 (2019) 717-731.

480 [13] Y. Zuo, J. Wang, W. Ma, Quasimodes instability analysis of uncertain asymmetric rotor system based on 3D solid element model, J. Sound Vib., 390 (2017) 192-204.

482 [14] I. Elishakoff, Y. Ren, Finite element methods for structures with large stochastic variations, Oxford 483 University Press Oxford, 2003.

484 [15] C. Soize, A nonparametric model of random uncertainties for reduced matrix models in structural 485 dynamics, Probabilist. Eng. Mech., 15 (2000) 277-294.

486 [16] R.E. Moore, Methods and Applications of Interval Analysis, SIAM, 1979.

487 [17] D. Moens, M. Hanss, Non-probabilistic finite element analysis for parametric uncertainty treatment in 488 applied mechanics: Recent advances, Finite Elem. Anal. Des., 47 (2011) 4-16.

489 [18] E.H. Koroishi, A.A. Cavalini Jr, A.M. de Lima, V. Steffen Jr, Stochastic modeling of flexible rotors, J. 490 Braz. Soc. Mecha. Sci., 34 (2012) 574-583.

491 [19] C. Fu, X. Ren, Y. Yang, K. Lu, W. Qin. Steady-state response analysis of cracked rotors with uncertain492 but-bounded parameters using a polynomial surrogate method. Commun. Nonlinear Sci. Numer. Simul., 68 493 (2019) 240-256.

494 [20] R. Murthy, J.C. Tomei, X.Q. Wang, M.P. Mignolet, A. El-Shafei, Nonparametric stochastic modeling of 495 ztructural uncertainty in rotordynamics: Unbalance and balancing sspects, J. Eng. Gas Turb. Power, 136 496 (2014) 062506.

497 [21] C. Gan, Y. Wang, S. Yang, Y. Cao, Nonparametric modeling and vibration analysis of uncertain Jeffcott 498 rotor with disc offset, Int. J. Mech. Sci., 78 (2014) 126-134.

499 [22] T.G. Ritto, R.H. Lopez, R. Sampaio, J.E.S.D. Cursi, Robust optimization of a flexible rotor-bearing 500 system using the Campbell diagram, Eng. Optimiz., 43 (2011) 77-96.

501 [23] H. Liao, Global resonance optimization analysis of nonlinear mechanical systems: Application to the uncertainty quantification problems in rotor dynamics, Commun. Nonlinear Sci. Numer. Simul., 19 (2014) 503 3323-3345.

504 [24] B. Liu, X. Yin, K. Jian, Y. Wu, Perturbation transfer matrix method for eigendata of one-dimensional structural system with parameter uncertainties, Appl. Math. Mech. Engl., 24 (2003) 801-807.

506 [25] J. Didier, B. Faverjon, J.J. Sinou, Analyzing the dynamic response of a rotor system under uncertain parameters by Polynomial Chaos Expansion, J. Vib. Control, 18 (2012) 587-607.

508 [26] E. Jacquelin, S. Adhikari, J.J. Sinou, M.I. Friswell, Polynomial chaos expansion in structural dynamics:

509 Accelerating the convergence of the first two statistical moment sequences, J. Sound Vib., 356 (2015) 144510154.

511 [27] J.J. Sinou, J. Didier, B. Faverjon, Stochastic non-linear response of a flexible rotor with local non512 linearities, Int. J. Nonlin.Mech., 74 (2015) 92-99.

513 [28] J.J. Sinou, E. Jacquelin, Influence of Polynomial Chaos expansion order on an uncertain asymmetric 514 rotor system response, Mech. Syst. Signal Pr., 50-51 (2015) 718-731.

515 [29] G. Alefeld, G. Mayer, Interval analysis: theory and applications, J. Comput. Appli. Math., 121 (1999) $516 \quad 421-464$.

517 [30] Z. Qiu, X. Wang, Several solution methods for the generalized complex eigenvalue problem with 518 bounded uncertainties, Int. J. Solids Struct., 42 (2005) 2883-2900.

519 [31] J. Wu, Y. Zhang, L. Chen, Z. Luo, A Chebyshev interval method for nonlinear dynamic systems under 520 uncertainty, Appl. Math. Model., 37 (2013) 4578-4591. 
[32] J. Wu, Z. Luo, N. Zhang, Y. Zhang, A new interval uncertain optimization method for structures using Chebyshev surrogate models, Comput. Struct., 146 (2015) 185-196.

[33] Y. Ma, Z. Liang, M. Chen, J. Hong, Interval analysis of rotor dynamic response with uncertain parameters, J. Sound Vib., 332 (2013) 3869-3880.

[34] C. Wang, Y. Ma, D. Zhang, J. Hong, Interval analysis on aero-engine rotor system with misalignment, ASME Turbo Expo 2015: Turbine Technical Conference and Exposition, American Society of Mechanical Engineers, 2015, V07AT30A002.

[35] C. Fu, X. Ren, Y. Yang, W. Qin, Dynamic response analysis of an overhung rotor with interval uncertainties, Nonlinear Dynam., 89 (2017) 2115-2124.

[36] K.A.F. Moustafa, H. El-Awady, Interval rotor-bearing systems stability via Monte Carlo simulation, Int. J. Modell. Simul., 28 (2008) 202-208.

[37] C. Fu, X. Ren, Y. Yang, Y. Xia, W. Deng, An interval precise integration method for transient unbalance response analysis of rotor system with uncertainty, Mech. Syst. Signal Pr., 107 (2018) 137-148.

[38] C. Jiang, J. Zheng, X. Han, Probability-interval hybrid uncertainty analysis for structures with both aleatory and epistemic uncertainties: a review, Struct. Multidiscip. Optimiz., 57 (2017) 2485-2502.

[39] B. Xia, D. Yu, J. Liu, Hybrid uncertain analysis for structural-acoustic problem with random and interval parameters, J. Sound Vib., 332 (2013) 2701-2720.

[40] D. Wu, W. Gao, Hybrid uncertain static analysis with random and interval fields, Comput. Methods Appl. Mech. Eng., 315 (2017) 222-246.

[41] J. Wu, Z. Luo, N. Zhang, Y. Zhang, A new uncertain analysis method and its application in vehicle dynamics, Mech. Syst. Signal Pr., 50-51 (2015) 659-675.

[42] M. Xu, J. Du, C. Wang, Y. Li, Hybrid uncertainty propagation in structural-acoustic systems based on the polynomial chaos expansion and dimension-wise analysis, Comput. Method. Appl. Mech. Eng., 320 (2017) 198-217.

[43] X. Du, A. Sudjianto, B. Huang, Reliability-based design with the mixture of random and interval variables, J. Mech. Des., 127 (2005) 1068-1076.

[44] E. Jacquelin, M.I. Friswell, S. Adhikari, O. Dessombz, J.J. Sinou, Polynomial chaos expansion with random and fuzzy variables, Mech. Syst. Signal Pr., 75 (2016) 41-56.

[45] L. Yang, J. Zhang, Y. Guo, Uncertainty representation and quantification for a nonlinear rotor/stator system with mixed uncertainties, J. Vibroeng., 18 (2016) 4836-4851.

[46] Y. Zhong, Y. He, Z. Wang, F. Li, Rotordynamics, Tsinghua University Press, 1987.

[47] T. Yamamoto, Y. Ishida, Linear and nonlinear rotordynamics: a modern treatment with applications, John Wiley \& Sons, 2001.

[48] Z. Luo, Y. Zhu, H. Liu, D. Wang, Dynamic similitude design method of the distorted model on variable thickness cantilever plates, Appl. Sci., 6 (2016) 228.

[49] F. Xie, H. Ma, C. Cui, B. Wen, Vibration response comparison of twisted shrouded blades using different impact models, J. Sound Vib., 397 (2017) 171-191.

[50] R.G. Ghanem, P.D. Spanos, Stochastic Finite Elements: A Spectral Approach, Courier Corporation, 2003.

[51] M. Loève, Probability Theory, Springer-Verlag, New York, 1977.

[52] Isukapalli SS. Uncertainty Analysis of Transport-Transformation Models. The State University of New Jersey, New Brunswick, 1999.

[53] H.A.P. da Silva, R. Nicoletti, Design of tilting-pad journal bearings considering bearing clearance uncertainty and reliability analysis. J. Tribol., 141 (2019) 011703. 
565 [54] Z. Zheng, Y. Xie, D. Zhang, Numerical investigation on the gravity response of a two-pole generator 566 rotor system with interval uncertainties, Appl. Sci., 9 (2019) 3036.

567 [55] C. Fu, X. Ren, Y. Yang, Vibration analysis of rotors under uncertainty based on Legendre series, J. Vib. 568 Eng. Technol., 7 (2019) 43-51. 